\title{
A NOTE ON QUASI-SIMILARITY OF KOOPMAN OPERATORS
}

\author{
K. FRĄCZEK AND M. LEMAŃCZYK
}

\begin{abstract}
Answering a question of A. Vershik we construct two non-weakly isomorphic ergodic automorphisms for which the associated unitary (Koopman) representations are Markov quasi-similar. We also discuss metric invariants of Markov quasi-similarity in the class of ergodic automorphisms.
\end{abstract}

\section{INTRODUCTION}

Markov operators appear in the classical ergodic theory in the context of joinings, see the monograph 77. Indeed, assume that $T_{i}$ is an ergodic automorphism of a standard probability Borel space $\left(X_{i}, \mathcal{B}_{i}, \mu_{i}\right), i=1,2$. Consider $\lambda$ a joining of $T_{1}$ and $T_{2}$, i.e. a $T_{1} \times T_{2}$-invariant probability measure on $\left(X_{1} \times X_{2}, \mathcal{B}_{1} \otimes \mathcal{B}_{2}\right)$ with the marginals $\mu_{1}$ and $\mu_{2}$ respectively. Then the operator $\Phi_{\lambda}: L^{2}\left(X_{1}, \mathcal{B}_{1}, \mu_{1}\right) \rightarrow$ $L^{2}\left(X_{2}, \mathcal{B}_{2}, \mu_{2}\right)$ determined by

$$
\left\langle\Phi_{\lambda} f_{1}, f_{2}\right\rangle_{L^{2}\left(X_{2}, \mathcal{B}_{2}, \mu_{2}\right)}=\left\langle f_{1} \otimes \mathbf{1}_{X_{2}}, \mathbf{1}_{X_{1}} \otimes f_{2}\right\rangle_{L^{2}\left(X_{1} \times X_{2}, \mathcal{B}_{1} \otimes \mathcal{B}_{2}, \lambda\right)}
$$

is Markov (i.e. it is a linear contraction which preserves the cone of non-negative functions and $\Phi_{\lambda} \mathbf{1}=\mathbf{1}=\Phi_{\lambda}^{*} \mathbf{1}$ ) and moreover

$$
\Phi_{\lambda} \circ U_{T_{1}}=U_{T_{2}} \circ \Phi_{\lambda},
$$

where $U_{T_{i}}: L^{2}\left(X_{i}, \mathcal{B}_{i}, \mu_{i}\right) \rightarrow L^{2}\left(X_{i}, \mathcal{B}_{i}, \mu_{i}\right)$ stands for the associated unitary operator: $U_{T_{i}} f=f \circ T_{i}$ for $f \in L^{2}\left(X_{i}, \mathcal{B}_{i}, \mu_{i}\right), i=1,2$, which is often called a Koopman operator. In fact, each Markov operator $\Phi: L^{2}\left(X_{1}, \mathcal{B}_{1}, \mu_{1}\right) \rightarrow L^{2}\left(X_{2}, \mathcal{B}_{2}, \mu_{2}\right)$ satisfying the equivariance property (2) is of the form $\Phi_{\lambda}$ for a unique joining $\lambda$ of $T_{1}$ and $T_{2}$ (see e.g. [17], [23]). Markov operators corresponding to ergodic joinings are called indecomposable.

In order to classify dynamical systems one usually considers the measure-theoretic isomorphism, i.e. the equivalence given by the existence of an invertible map $S$ : $\left(X_{1}, \mathcal{B}_{1}, \mu_{1}\right) \rightarrow\left(X_{2}, \mathcal{B}_{2}, \mu_{2}\right)$ for which $S \circ T_{1}=T_{2} \circ S$. The measure-theoretic (metric) isomorphism implies spectral equivalence of the Koopman operators $U_{T_{1}}$ and $U_{T_{2}}$; indeed, $U_{S^{-1}}$ (where $U_{S^{-1}} f_{1}=f_{1} \circ S^{-1}$ for $f_{1} \in L^{2}\left(X_{1}, \mathcal{B}_{1}, \mu_{1}\right)$ ) provides such an equivalence. The converse does not hold, see e.g. [1]; we also recall that all Bernoulli shifts are spectrally equivalent while the entropy classify them measure-theoretically [19]. One may ask whether there can be some other natural classification of dynamical systems which lies in between metric and spectral equivalence.

In [25], A. Vershik considers the quasi-similarity problem in the class of Markov operators. Recall that if $A_{i}$ is a bounded linear operator of a Hilbert space $H_{i}$, $i=1,2$, and if there is a bounded linear operator $V: H_{1} \rightarrow H_{2}$ whose range is

2000 Mathematics Subject Classification. 37A05, 37A30, 37A35.

Research partially supported by Polish MNiSzW grant N N201 384834; partially supported by Marie Curie "Transfer of Knowledge" EU program - project MTKD-CT-2005-030042 (TODEQ). 
dense and which intertwines $A_{1}$ and $A_{2}$, then $A_{2}$ is said to be a quasi-image of $A_{1}$. By duality, $A_{2}$ is a quasi-image of $A_{1}$ if and only if there exists a $1-1$ bounded linear operator $W: H_{2} \rightarrow H_{1}$ intertwining $A_{2}$ and $A_{1}$. If also $A_{1}$ is a quasi-image of $A_{2}$ then the two operators are called quasi-similar. The main problem taken up in [25] is a study of quasi-similarity in the class of Markov operators, i.e. given a Markov operator $A_{i}$ of $H_{i}=L^{2}\left(X_{i}, \mathcal{B}_{i}, \mu_{i}\right), i=1,2$, we investigate a possible quasi-similarity of $A_{1}$ and $A_{2}$, where we additionally require $V$ to be a Markov operator between the corresponding $L^{2}$-spaces. In what follows, we will call such a quasi-similarity Markov quasi-similarity.

Notice that each Koopman operator is also a Markov operator. It is known (see e.g. [15, 25]) that if an intertwining Markov operator $\Phi: L^{2}\left(X_{1}, \mathcal{B}_{1}, \mu_{1}\right) \rightarrow$ $L^{2}\left(X_{2}, \mathcal{B}_{2}, \mu_{2}\right)$ is unitary then it has to be of the form $U_{S}$ where $S$ provides a measure-theoretic isomorphism. On the other hand the quasi-similarity of unitary operators implies their spectral equivalence (see Section 1 below). Therefore, Markov quasi-similarity lies in between the spectral and measure-theoretic equivalence of dynamical systems. One of questions raised by Vershik in [25] is the following:

\section{Do there exist two automorphisms that are not isomorphic but are Markov quasi-similar?}

In order to answer this question notice that any weakly isomorphic automorphisms (see [24]) $T_{1}$ and $T_{2}$ are automatically Markov quasi-similar; indeed, the weak isomorphism means that there are $\pi_{1}$ and $\pi_{2}$ which are homomorphisms between $T_{1}$ and $T_{2}$ and $T_{2}$ and $T_{1}$ respectively, then $U_{\pi_{1}}^{*}$ and $U_{\pi_{2}}^{*}$ yield Markov quasi-similarity of $T_{1}$ and $T_{2}$. Hence, if $T_{1}$ and $T_{2}$ are weakly isomorphic but not isomorphic, we obtain the positive answer to the question (3). Examples of weakly isomorphic but not isomorphic systems are known in the literature, see e.g. [12], [13, [22], including the case of K-automorphisms [8]. It follows that the notion of Markov quasi-similarity has to be considered as an interesting refinement of the notion of weak isomorphism, and in Vershik's question (3) we have to replace "not isomorphic" by "not weakly isomorphic".

The main aim of this note is to answer positively this modified question (3) (see Proposition 7 below). We would like to emphasize that despite a spectral flavor of the definition, Markov quasi-similarity is far from being the same as spectral equivalence. For example, partly answering Vershik's question raised at a seminar at Penn State University in 2004 whether entropy is an invariant of Markov quasisimilarity, we show that zero entropy as well as K-property are invariants of Markov quasi-similarity of automorphisms, while they are not invariants of spectral equivalence of the corresponding unitary operators. These facts and related problems will be discussed in Sections 4,6 ,

\section{QUASI-SIMILARITY OF UNITARY OPERATORS IMPLIES THEIR UNITARY EQUIVALENCE}

Assume that $U$ is a unitary operator of a separable Hilbert space $H$. Given $x \in H$ by $\mathbb{Z}(x)$ we denote the cyclic space generated by $x$, i.e. $\mathbb{Z}(x)=\overline{\operatorname{span}}\left\{U^{n} x: n \in \mathbb{Z}\right\}$. We will use a similar notation $\mathbb{Z}\left(y_{1}, \ldots, y_{k}\right)$ for the smallest closed $U$-invariant subspace containing $y_{i}, i=1, \ldots, k$. Denote by $\mathbb{T}$ the (additive) circle. Then the Fourier transform of the (positive) measure $\sigma_{x}$ - called the spectral measure of $x-$ 
is given by

$$
\widehat{\sigma}_{x}(n):=\int_{\mathbb{T}} e^{2 \pi i n t} d \sigma_{x}(t)=\left\langle U^{n} x, x\right\rangle \text { for each } n \in \mathbb{Z} .
$$

Similarly the sequence $\left(\left\langle U^{n} x, y\right\rangle\right)_{n \in \mathbb{Z}}$ is the Fourier transform of the (complex) spectral measure $\sigma_{x, y}$ of $x$ and $y$. Given a spectral measure $\sigma$ we denote

$$
H_{\sigma}=\left\{x \in H: \sigma_{x} \ll \sigma\right\} .
$$

Then $H_{\sigma}$ is a closed $U$-invariant subspace called a spectral subspace of $H$.

It follows from Spectral Theorem for unitary operators (see e.g. [1] or 20]) that there is a decomposition

$$
H=H_{\sigma_{1}} \oplus H_{\sigma_{2}} \oplus \ldots
$$

into spectral subspaces such that for each $i \geq 1$

$$
H_{\sigma_{i}}=\bigoplus_{k=1}^{n_{i}} \mathbb{Z}\left(x_{k}^{(i)}\right),
$$

where $\sigma_{i} \equiv \sigma_{x_{1}^{(i)}} \equiv \sigma_{x_{2}^{(i)}} \equiv \ldots\left(n_{i}\right.$ can be infinity), and $\sigma_{i} \perp \sigma_{j}$ for $i \neq j$. The class $\sigma_{U}$ of all finite measures equivalent to the sum $\sum_{i \geq 1} \sigma_{i}$ is then called the maximal spectral type of $U$. Another important invariant of $U$ is the spectral multiplicity function $M_{U}: \mathbb{T} \rightarrow\{1,2, \ldots\} \cup\{\infty\}$ (see [11, [20]) which is defined $\sigma$-a.e., where $\sigma$ is any measure belonging to the maximal spectral type of $U$. Note that decomposition (4) is far from being unique but if

$$
H=\bigoplus_{i=1}^{\infty} H_{\sigma_{i}^{\prime}}, \quad H_{\sigma_{i}^{\prime}}=\bigoplus_{k=1}^{n_{i}^{\prime}} \mathbb{Z}\left(y_{k}^{(i)}\right)
$$

is another decomposition (4) in which $\sigma_{i} \equiv \sigma_{i}^{\prime}, i \geq 1$, then $n_{i}=n_{i}^{\prime}$ for $i \geq 1$. Recall that the essential supremum $m_{U}$ of $M_{U}$ (called the maximal spectral multiplicity of $U)$ is equal to

$$
\inf \left\{m \geq 1: \mathbb{Z}\left(y_{1}, \ldots, y_{m}\right)=H \text { for some } y_{1}, \ldots, y_{m} \in H\right\} ;
$$

if there is no "good" $m$, them $m_{U}=\infty$.

Assume that $U_{i}$ is a unitary operator of a separable Hilbert space $H_{i}, i=1,2$. Let $V: H_{1} \rightarrow H_{2}$ be a bounded linear operator which intertwines $U_{1}$ and $U_{2}$. Then for each $n \in \mathbb{Z}$ and $x_{1} \in H_{1}$

$$
\left\langle U_{2}^{n} V x_{1}, V x_{1}\right\rangle=\left\langle U_{1}^{n} x_{1}, V^{*} V x_{1}\right\rangle,
$$

so by elementary properties of spectral measures

$$
\sigma_{V x_{1}}=\sigma_{x_{1}, V^{*} V x_{1}} \ll \sigma_{x_{1}} .
$$

Assuming additionally that $\operatorname{Im}(V)$ is dense, an immediate consequence of (6) is that the maximal spectral type of a quasi-image of $U_{1}$ is absolutely continuous with respect to $\sigma_{U_{1}}$. It is also clear that given $y_{1}^{(1)}, \ldots, y_{m}^{(1)} \in H_{1}$ we have

$$
\overline{V\left(\mathbb{Z}\left(y_{1}^{(1)}, \ldots, y_{m}^{(1)}\right)\right)}=\mathbb{Z}\left(V y_{1}^{(1)}, \ldots, V y_{m}^{(1)}\right) .
$$

This in turn implies that the maximal spectral multiplicity of a quasi-image of $U_{1}$ is at most $m_{U_{1}}$.

Proposition 1. If $U_{1}$ and $U_{2}$ are quasi-similar then they are spectrally equivalent. 
Proof. Assume that $V: H_{1} \rightarrow H_{2}$ and $W: H_{2} \rightarrow H_{1}$ intertwine $U_{1}$ and $U_{2}$ and have dense ranges. In view of (6) both operators $U_{1}$ and $U_{2}$ have the same maximal spectral types. Consider a decomposition (4) for $U_{1}: H_{1}=\bigoplus_{i \geq 1} H_{\sigma_{i}^{(1)}}$ and let $F_{i}:=\overline{V\left(H_{\sigma_{i}^{(1)}}\right)}$ for $i \geq 1$. The subspaces $F_{i}$ are obviously $U_{2}$-invariant and let $\sigma_{i}^{(2)}$ $\left(n_{i}^{(2)}\right)$ denote the maximal spectral type (the maximal spectral multiplicity) of $U_{2}$ on $F_{i}$. It follows from (6) that $\sigma_{i}^{(2)} \ll \sigma_{i}^{(1)}$ for $i \geq 1$ and $\sigma_{i}^{(2)}, \sigma_{j}^{(2)}$ are mutually singular (in particular, $F_{i} \perp F_{j}$ ) whenever $i \neq j$. Moreover, $n_{i}^{(2)} \leq n_{i}^{(1)}, i \geq 1$. Since $V$ has dense range, $H_{2}=\bigoplus_{i \geq 1} F_{i}$. It follows that (up to equivalence of measures) $\sum_{i \geq 1} \sigma_{2}^{(i)}$ is the maximal spectral type of $U_{2}$ hence it is equivalent to $\sum_{i \geq 1} \sigma_{i}^{(1)}$ and therefore $\sigma_{i}^{(1)} \equiv \sigma_{i}^{(2)}$ for $i \geq 1$. The same reasoning applied to the decomposition $H_{2}=\bigoplus_{i \geq 1} F_{i}$ and $W$ shows that $H_{1}=\bigoplus_{i \geq 1} \overline{W\left(F_{i}\right)}$ and the maximal spectral type of $U_{1}$ on $\overline{W\left(F_{i}\right)}$ is absolutely continuous with respect to $\sigma_{i}^{(2)} \equiv \sigma_{i}^{(1)}, i \geq 1$. It follows that $\overline{W\left(F_{i}\right)}=H_{\sigma_{i}^{(1)}}$ for all $i \geq 1$. In particular, we have proved that $n_{i}^{(2)}=n_{i}^{(1)}$ but we need to show that on $F_{i}$ the multiplicity is uniform. Suppose this is not the case, i.e. that for some measure $\eta \ll \sigma_{i}^{(2)}$ we have

$$
F_{i}=\mathbb{Z}\left(z_{1}\right) \oplus \ldots \oplus \mathbb{Z}\left(z_{r}\right) \oplus F_{i}^{\prime},
$$

where for $j=1, \ldots r, \sigma_{z_{j}}=\eta, 1 \leq r<n_{i}^{(2)}$ and the maximal spectral type of $U_{2}$ on $F_{i}^{\prime}$ is orthogonal to $\eta$. We have

$$
H_{\sigma_{i}^{(1)}}=\overline{W\left(F_{i}\right)}=G_{i} \oplus \overline{W\left(F_{i}^{\prime}\right)}
$$

where $G_{i}=\overline{W\left(\mathbb{Z}\left(z_{1}\right) \oplus \ldots \oplus \mathbb{Z}\left(z_{r}\right)\right)}$ and the maximal spectral types on $G_{i}$, say $\tau(\ll \eta)$, and $\overline{W\left(F_{i}^{\prime}\right)}$ are mutually singular. It follows that the multiplicity of $\tau$ is at most $r$, which is a contradiction since all measures absolutely continuous with respect to $\sigma_{i}^{(1)}$ have multiplicity $n_{i}^{(1)}$.

Remark 1. Literally speaking, the notion of quasi-similarity is weaker than the classical notion of quasi-affinity [4]: $A_{1}$ and $A_{2}$ are quasi-affine if there exists a $1-1$ bounded linear operator $V: H_{1} \rightarrow H_{2}$ with dense range intertwining $A_{1}$ and $A_{2}$. Proposition 3.4 in [4] tells us that quasi-affine unitary operators are unitarily equivalent. Hence Proposition 1 shows in fact that for unitary operators quasisimilarity and quasi-affinity are equivalent notions.

Similarly to Markov quasi-similarity of Koopman operators we can speak about their Markov quasi-affinity. It is not clear (see Section 6) whether these two notions coincide.

\section{A Convolution operator in $l^{2}(\mathbb{Z})$}

In this section we produce a sequence in $l^{2}(\mathbb{Z})$ which will be used to construct a Markov quasi-affinity between two non-weakly isomorphic automorphisms in Section 3 .

Denote by $l_{0}(\mathbb{Z})$ the subspace of $l^{2}(\mathbb{Z})$ of complex sequences $\bar{x}=\left(x_{n}\right)_{n \in \mathbb{Z}}$ such that $\left\{n \in \mathbb{Z}: x_{n} \neq 0\right\}$ is finite. 
Proposition 2. There exists a nonnegative sequence $\bar{a}=\left(a_{n}\right)_{n \in \mathbb{Z}} \in l^{2}(\mathbb{Z})$ such that $\sum_{n \in \mathbb{Z}} a_{n}=1$ and

$$
\text { for every } \bar{x}=\left(x_{n}\right)_{n \in \mathbb{Z}} \in l^{2}(\mathbb{Z}) \text { if } \bar{a} * \bar{x} \in l_{0}(\mathbb{Z}) \text { then } \bar{x}=\overline{0} .
$$

Each element $\bar{y} \in l^{2}(\mathbb{Z})$ is an $L^{2}$-function on $\mathbb{Z}$ and its Fourier transform is a function $h \in L^{2}(\mathbb{T})$ for which $\widehat{h}(n)=y_{n}$ for all $n \in \mathbb{Z}$. Moreover, the convolution of $l^{2}$-sequences corresponds to the pointwise multiplication of $L^{2}$-functions on the circle. It follows that in order to find the required sequence $\bar{a}$, it suffices to find a function $f \in L^{2}(\mathbb{T})$ such that

- $a_{n}=\hat{f}(n) \geq 0, \sum_{n \in \mathbb{Z}} a_{n}=1$;

- for every $g \in L^{2}(\mathbb{T})$, if $f \cdot g=0$ then $g=0$;

- for every non-zero trigonometric polynomial $P$, if $P=f \cdot g$ then $g \notin L^{2}(\mathbb{T})$.

This is done below.

Lemma 3. If $f:[0,1] \rightarrow \mathbb{R}_{+}$is a convex $C^{2}$-function such that $f(1-x)=f(x)$ for all $x \in[0,1]$ then $\hat{f}(n) \geq 0$ for all $n \in \mathbb{Z}$.

Proof. By assumption, $f^{\prime \prime}(x) \geq 0$ for all $x \in[0,1]$. Using integration by parts twice, for $n \neq 0$ we obtain

$$
\begin{aligned}
\hat{f}(n) & =\int_{0}^{1} f(x) e^{-2 \pi i n x} d x=\int_{0}^{1} f(x) \cos (2 \pi n x) d x=\frac{1}{2 \pi n} \int_{0}^{1} f(x) d \sin (2 \pi n x) \\
& =-\frac{1}{2 \pi n} \int_{0}^{1} f^{\prime}(x) \sin (2 \pi n x) d x=\frac{1}{4 \pi^{2} n^{2}} \int_{0}^{1} f^{\prime}(x) d \cos (2 \pi n x) \\
& =\frac{1}{4 \pi^{2} n^{2}}\left[f^{\prime}(1)-f^{\prime}(0)-\int_{0}^{1} f^{\prime \prime}(x) \cos (2 \pi n x) d x\right] \\
& \geq \frac{1}{4 \pi^{2} n^{2}}\left[f^{\prime}(1)-f^{\prime}(0)-\int_{0}^{1}\left|f^{\prime \prime}(x) \cos (2 \pi n x)\right| d x\right] \\
& \geq \frac{1}{4 \pi^{2} n^{2}}\left[f^{\prime}(1)-f^{\prime}(0)-\int_{0}^{1} f^{\prime \prime}(x) d x\right]=0 .
\end{aligned}
$$

Proof of Proposition Q Q Let us consider $f:[0,1] \rightarrow \mathbb{R}$ defined by

$$
f(x)=\left\{\begin{array}{ccc}
e^{-\frac{1}{|x-1 / 2|}+2} & \text { if } & x \neq 1 / 2 \\
0 & \text { if } & x=1 / 2
\end{array}\right.
$$

Since $f^{\prime \prime}(x) \geq 0$ for $x \in[0,1]$, by Lemma [3, $a_{n}=\hat{f}(n) \geq 0$. As $f: \mathbb{T} \rightarrow \mathbb{R}$ is a continuous function of bounded variation,

$$
1=f(0)=\sum_{n \in \mathbb{Z}} a_{n}
$$

Since $f(x) \neq 0$ for $x \neq 12$, if $f \cdot g=0$ for some $g \in L^{2}(\mathbb{T})$ then $g=0$.

Suppose, contrary to our claim, that there exist $g \in L^{2}(\mathbb{T})$ and a non-zero trigonometric polynomial $P$ such that $f \cdot g=P$. Recall that for every $m \geq 0$ we have $\int_{0}^{1} e^{1 / x} x^{m} d x=+\infty$, hence $\int_{0}^{1}\left(e^{1 / x} x^{m}\right)^{2} d x=+\infty$. Since $P$ is a non-zero analytic function, there exists $m \geq 0$ such that $P^{(m)}(1 / 2) \neq 0$ and $P^{(k)}(1 / 2)=0$ 
for $0 \leq k<m$. By Taylor's formula, there exist $C>0$ and $0<\delta<1 / 2$ such that $|P(x+1 / 2)| \geq C|x|^{m}$ for $x \in[-\delta, \delta]$. It follows that

$$
\begin{aligned}
\int_{\mathbb{T}}|g(x)|^{2} d x & \geq \int_{1 / 2}^{1 / 2+\delta}|P(x)|^{2} / f(x)^{2} d x=\int_{0}^{\delta}|P(x+1 / 2)|^{2} / f(x+1 / 2)^{2} d x \\
& \geq \int_{0}^{\delta}\left(C x^{m} e^{1 / x}\right)^{2} d x=+\infty
\end{aligned}
$$

and hence $g \notin L^{2}(\mathbb{T})$ which completes the proof.

\section{TWO NON-WEAKLY ISOMORPHIC AUTOMORPHISMS WHICH ARE MARKOV QUASI-SIMILAR}

Let $T$ be an ergodic automorphism of $(X, \mathcal{B}, \mu)$. Assume that $G$ is a compact metric Abelian group with Haar measure $\lambda_{G}$. A measurable function $\varphi: X \rightarrow G$ is called a cocycle. Using the cocycle we can define a group extension $T_{\varphi}$ of $T$ which acts on $\left(X \times G, \mathcal{B} \otimes \mathcal{B}(G), \mu \otimes \lambda_{G}\right)$ by the formula $T_{\varphi}(x, g)=(T x, \varphi(x)+g)$.

We will first take $\varphi: X \rightarrow \mathbb{Z}_{2}:=\{0,1\}$ so that the group extension $T_{\varphi}$ is ergodic. Then assume that we can find $S$ acting on $(X, \mathcal{B}, \mu), S T=T S$, such that if we put $G=\mathbb{Z}_{2}^{\mathbb{Z}}$ and define

$$
\psi: X \rightarrow G, \psi(x)=\left(\ldots, \varphi\left(S^{-1} x\right), \stackrel{0}{(x)}(x), \varphi(S x), \varphi\left(S^{2} x\right), \ldots\right)
$$

then $T_{\psi}$ is ergodic as well (see [13 for concrete examples of $T, \varphi$ and $S$ fulfilling our requirements). Put now $T_{1}=T_{\psi}$ and let us take a factor $T_{2}$ of $T_{1}$ obtained by "forgeting" the first $\mathbb{Z}_{2}$-coordinate. In other words on $\left(X \times \mathbb{Z}_{2}^{\mathbb{Z}}, \mu \otimes \lambda_{\mathbb{Z}_{2}^{\mathbb{Z}}}\right)$ we consider two automorphisms

$$
\begin{aligned}
& T_{1}(x, \underline{i})=\left(T x, \ldots, i_{-1}+\varphi\left(S^{-1} x\right), i_{0}+\varphi(x), i_{1}+\varphi(S x), i_{2}+\varphi\left(S^{2} x\right), \ldots\right), \\
& T_{2}(x, \underline{i})=\left(T x, \ldots, i_{-1}+\varphi\left(S^{-1} x\right), i_{0}+\varphi(x), i_{1}+\varphi\left(S^{2} x\right), i_{2}+\varphi\left(S^{3} x\right), \ldots\right),
\end{aligned}
$$

where $\underline{i}=\left(\ldots, i_{-1}, \stackrel{0}{i_{0}}, i_{1}, i_{2}, \ldots\right)$. Define $I_{n}: X \times \mathbb{Z}_{2}^{\mathbb{Z}} \rightarrow X \times \mathbb{Z}_{2}^{\mathbb{Z}}$ by putting

$$
I_{n}(x, \underline{i})=\left(S^{n} x, \ldots, i_{n-1}, i_{n}, i_{n+2}, i_{n+3}, \ldots\right) .
$$

Then $I_{n}$ is measure-preserving and $I_{n} \circ T_{1}=T_{2} \circ I_{n}$. Therefore

$$
U_{T_{1}} \circ U_{I_{n}}=U_{I_{n}} \circ U_{T_{2}}
$$

with $U_{I_{n}}$ being an isometry (which is not onto) and

$$
\begin{aligned}
& U_{I_{n}}^{*} F(x, \underline{i}) \\
& \quad=\frac{1}{2}\left(F\left(S^{-n} x, \ldots, i_{-n}^{0}, \ldots, i_{0}, 0, i_{1}, \ldots\right)+F\left(S^{-n} x, \ldots, i_{-n}^{0}, \ldots, i_{0}, 1, i_{1}, \ldots\right)\right) .
\end{aligned}
$$

Let $\bar{a}=\left(a_{n}\right)_{n \in \mathbb{Z}} \in l^{2}(\mathbb{Z})$ be a nonnegative sequence such that $\sum_{n \in \mathbb{Z}} a_{n}=1$ and (7) holds. Let $J: L^{2}\left(X \times \mathbb{Z}_{2}^{\mathbb{Z}}, \mu \otimes \lambda_{\mathbb{Z}_{2}^{\mathbb{Z}}}\right) \rightarrow L^{2}\left(X \times \mathbb{Z}_{2}^{\mathbb{Z}}, \mu \otimes \lambda_{\mathbb{Z}_{2}^{\mathbb{Z}}}\right)$ stand for the Markov operator defined by

$$
J=\sum_{n \in \mathbb{Z}} a_{n} U_{I_{n}} .
$$

In view of (8), $J$ intertwines $U_{T_{1}}$ and $U_{T_{2}}$. 
Denote by Fin the set of finite nonempty subsets of $\mathbb{Z}$. Let us consider two operations on Fin:

$$
\begin{gathered}
\widehat{A}=\{s \in A: s \leq 0\} \cup\{s+1: s \in A, s>0\} \text { for } A \in \text { Fin } \\
\widetilde{B}=\{s \in B: s \leq 0\} \cup\{s-1: s \in B, s>1\} \text { for } B \in \text { Fin with } 1 \notin B .
\end{gathered}
$$

Of course, $\widetilde{\widehat{A}}=A$ and $\widehat{\widetilde{B}}=B$. Let $\sim$ stand for the equivalence relation in Fin defined by $A \sim B$ if $A=B+n$ for some $n \in \mathbb{Z}$. Denote by $F i n_{0}$ a fundamental domain for this relation.

Lemma 4. $J$ has trivial kernel.

Proof. Each $F \in L^{2}\left(X \times \mathbb{Z}_{2}^{\mathbb{Z}}, \mu \otimes \lambda_{\mathbb{Z}_{2}^{\mathbb{Z}}}\right)$ can be written as

$$
F(x, \underline{i})=\sum_{A \in F i n} f_{A}(x)(-1)^{A(\underline{i})} \text {, where } A(\underline{i})=\sum_{s \in A} i_{s} .
$$

Note that $\sum_{A \in F i n}\left\|f_{A}\right\|_{L^{2}(X, \mu)}^{2}=\|F\|_{L^{2}\left(X \times \mathbb{Z}_{2}^{\mathbb{Z}}, \mu \otimes \lambda_{\mathbb{Z}_{2}^{\mathbb{Z}}}\right)}^{2}$. Since

$$
U_{I_{n}}\left(f_{A} \otimes(-1)^{A(\cdot)}\right)(x, \underline{i})=\left(f_{A} \otimes(-1)^{A(\cdot)}\right)\left(I_{n}(x, \underline{i})\right)=f_{A}\left(S^{n} x\right)(-1)^{(\widehat{A}+n)(\underline{i})},
$$

we have

$$
J F(x, \underline{i})=\sum_{n \in \mathbb{Z}} \sum_{A \in F i n} a_{n} f_{A}\left(S^{n} x\right)(-1)^{(\widehat{A}+n)(\underline{i})} .
$$

Notice that $n+1 \notin \widehat{A}+n$. To reverse the roles played by $A$ and $\widehat{A}+n$ note that if $B \in F$ in and $n+1 \notin B$ then the set $\widetilde{B-n}$ is the unique set such that $\widetilde{\widehat{B-n}}+n=B$. It follows that

$$
J F(x, \underline{i})=\sum_{B \in F i n} \sum_{n \in \mathbb{Z}, n+1 \notin B} a_{n} f_{\widetilde{B-n}}\left(S^{n} x\right)(-1)^{B(\underline{i})}=\sum_{B \in F i n} \widetilde{F}_{B}(x)(-1)^{B(\underline{i})},
$$

where $\widetilde{F}_{B}(x)=\sum_{n \in \mathbb{Z}, n+1 \notin B} a_{n} f_{\widetilde{B-n}}\left(S^{n} x\right)$. For every $B \in F i n_{0}$ and $x \in X$ we define $\xi^{B}(x)=\left(\xi_{n}^{B}(x)\right)_{n \in \mathbb{Z}}$ by setting

$$
\xi_{-n}^{B}(x)=\left\{\begin{array}{cll}
f_{\widetilde{B-n}}\left(S^{n} x\right) & \text { if } & n+1 \notin B \\
0 & \text { if } & n+1 \in B .
\end{array}\right.
$$

Therefore, for $k \in \mathbb{Z}$

$$
\begin{aligned}
\widetilde{F}_{B+k}(x) & =\sum_{n \in \mathbb{Z}, n+1 \notin B+k} a_{n} f_{\widetilde{B-n+k}}\left(S^{n} x\right) \\
& =\sum_{n \in \mathbb{Z},(n-k)+1 \notin B} a_{n} \widetilde{f_{B-(n-k)}}\left(S^{-(k-n)}\left(S^{k} x\right)\right) \\
& =\sum_{n \in \mathbb{Z}} a_{n} \xi_{k-n}^{B}\left(S^{k} x\right)=\left[\bar{a} *\left(\xi^{B}\left(S^{k} x\right)\right)\right]_{k} .
\end{aligned}
$$

Suppose that $J(F)=0$. It follows that given $k \in \mathbb{Z}$ and $B \in$ Fin $_{0}$ we have $\left[\bar{a} *\left(\xi^{B}\left(S^{k} x\right)\right)\right]_{k}=\widetilde{F}_{B+k}(x)=0$ for $\mu$-a.e. $x \in X$, whence a.s. we also have $[\bar{a} *$ $\left.\left(\xi^{B}(x)\right)\right]_{k}=0$. Letting $k$ run through $\mathbb{Z}$ we obtain that $\bar{a} *\left(\xi^{B}(x)\right)=\overline{0}$ for $\mu$-a.e. $x \in X$. On the other hand $\xi^{B}(x) \in l^{2}(\mathbb{Z})$ for almost every $x \in X$. In view of (7), $\xi^{B}(x)=\overline{0}$ for every $B \in$ Fin $_{0}$ and for a.e. $x \in X$, hence $f_{\widetilde{A}}=0$ for every $A \in$ Fin with $1 \notin A$. It follows that $f_{A}=0$ for every $A \in$ Fin, consequently $F=0$. 
Lemma 5. $J^{*}$ has trivial kernel.

Proof. Let

$$
F(x, \underline{i})=\sum_{A \in F i n} f_{A}(x)(-1)^{A(\underline{i})} .
$$

Then

$$
U_{I_{n}}^{*}\left(f_{A} \otimes(-1)^{A(\cdot)}\right)(x, \underline{i})=\left\{\begin{array}{ccc}
f_{A}\left(S^{-n} x\right)(-1)^{\widetilde{A-n}(\underline{i})} & \text { if } & n+1 \notin A \\
0 & \text { if } & n+1 \in A .
\end{array}\right.
$$

It follows that

$$
\begin{aligned}
J^{*} F(x, \underline{i}) & =\sum_{A \in \text { Fin }} \sum_{n \in \mathbb{Z}, n+1 \notin A} a_{n} f_{A}\left(S^{-n} x\right)(-1)^{\widetilde{A-n}(\underline{i})} \\
& =\sum_{B \in \text { Fin }} \sum_{n \in \mathbb{Z}} a_{n} f_{\widehat{B}+n}\left(S^{-n} x\right)(-1)^{B(\underline{i})} \\
& =\sum_{A \in \text { Fin }, 1 \notin A} \sum_{n \in \mathbb{Z}} a_{n} f_{A+n}\left(S^{-n} x\right)(-1)^{\widetilde{A}(\underline{i})} .
\end{aligned}
$$

Furthermore,

$$
\begin{aligned}
J^{*} F(x, \underline{i}) & =\sum_{A \in F i n_{0}} \sum_{k \in \mathbb{Z}, 1 \notin A-k} \sum_{n \in \mathbb{Z}} a_{n} f_{A+n-k}\left(S^{-n} x\right)(-1)^{\widetilde{A-k}(\underline{i})} \\
& =\sum_{A \in F i n_{0}} \sum_{k \in \mathbb{Z}, 1 \notin A-k}\left[\bar{a} *\left(\zeta^{A}\left(S^{-k} x\right)\right)\right]_{k}(-1)^{\widetilde{A-k}(\underline{i})},
\end{aligned}
$$

where $\zeta^{A}(x)=\left(\zeta^{A}(x)_{l}\right)_{l \in \mathbb{Z}}$ is given by $\zeta^{A}(x)_{l}=f_{A-l}\left(S^{l} x\right)$.

Suppose that $J^{*}(F)=0$. It follows that $\left[\bar{a} * \zeta^{A}\left(S^{-k} x\right)\right]_{k}=0$ for every $A \in$ Fin $_{0}$, $k+1 \notin A$ and for a.e. $x \in X$. Hence $\bar{a} *\left(\zeta^{A}(x)\right) \in l_{0}(\mathbb{Z})$ for $\mu$-a.e. $x \in X$ (the only possibly non-zero terms of the convolved sequence have indices belonging to $A-1)$. Since $\zeta^{A}(x) \in l^{2}(\mathbb{Z})$, in view of (7), $\zeta^{A}(x)=\overline{0}$ for every $A \in F i n_{0}$ and for $\mu$-a.e. $x \in X$. Thus $f_{A}=0$ for all $A \in F$ in and consequently $F=0$.

It follows from the above two lemmas that the ranges of $J$ and $J^{*}$ are dense. Clearly $J$ and $J^{*}$ intertwine the Koopman operators $U_{T_{1}}$ and $U_{T_{2}}$, hence we have proved the following.

Proposition 6. Under the above notation the automorphisms $T_{1}$ and $T_{2}$ are Markov quasi-similar.

Recall that in [13] constructions of the above type have been used to produce weakly isomorphic transformations that are not isomorphic. In fact our transformation $T_{1}$ is the same as the transformation $T_{\ldots,-1,0,1,2, \ldots}$ in Subsection 4.2 in [13, where it is proved that each metric endomorphism that commutes with $T_{1}$ is invertible. It follows that $T_{1}$ cannot be a factor of the system given by its proper factor; in particular, it is not weakly isomorphic to $T_{2}$. In other words we have proved the following.

Proposition 7. There are ergodic automorphisms which are Markov quasi-similar but they are not weakly isomorphic.

Remark 2. The Markov quasi-similarity between $T_{1}$ and $T_{2}$ constructed above is given by a $1-1$ Markov operator with dense range, that is, in fact we have shown that $U_{T_{1}}$ and $U_{T_{2}}$ are Markov quasi-affine. The Markov operator is given as a 
convex combination of isometries which separately have no dense ranges as they are not onto (and obviously their ranges are closed). Let us emphasize that not each non-trivial choice of weights $\left(a_{n}\right)$ gives rise to an operator with dense range as the following example shows.

Example. Set $a_{n}=\frac{1}{2^{n+1}}$ for $n \geq 0$ and $a_{n}=0$ for $n<0$. We will show that in this case $\operatorname{ker} J^{*} \neq\{0\}$. Denoting by $\bar{S}$ the automorphism of $\left(X \times \mathbb{Z}_{2}^{\mathbb{Z}}, \mu \otimes \lambda_{\mathbb{Z}_{2}^{\mathbb{Z}}}\right)$ given by

$$
\bar{S}(x, \underline{i})=\left(S x, \ldots, i_{-1}, i_{0}, \stackrel{0}{i_{1}}, i_{2}, \ldots\right),
$$

we have $I_{n}=I_{0} \circ \bar{S}^{n}$ for any $n \in \mathbb{Z}$, and hence

$$
J^{*}=U_{I_{0}}^{*} \circ \sum_{n=0}^{\infty} \frac{1}{2^{n+1}} U_{\bar{S}^{-n}} .
$$

In fact, we will prove that

$$
\left(-\frac{1}{2} U_{\bar{S}^{-1}}+I d\right)\left(\operatorname{ker} U_{I_{0}}^{*}\right) \subset k e r J^{*} .
$$

Notice that if $0 \neq G \in L^{2}\left(X \times \mathbb{Z}_{2}^{\mathbb{Z}}, \mu \otimes \lambda_{\mathbb{Z}_{2}}^{\mathbb{Z}}\right)$ then $-\frac{1}{2} G \circ \bar{S}^{-1}+G \neq 0$ because the norms of the two summands are different. To prove (9) take $G \in \operatorname{ker} U_{I_{0}}^{*}$ and let $F=-\frac{1}{2} G \circ \bar{S}^{-1}+G$. Thus

$$
\begin{aligned}
J^{*} F & =U_{I_{0}}^{*}\left(\sum_{n=0}^{\infty} \frac{1}{2^{n+1}} F \circ \bar{S}^{-n}\right) \\
& =U_{I_{0}}^{*} \sum_{n=0}^{\infty}\left(\frac{1}{2^{n+1}} G \circ \bar{S}^{-n}-\frac{1}{2^{n+2}} G \circ \bar{S}^{-n-1}\right)=U_{I_{0}}^{*}\left(\frac{1}{2} G\right)=0 .
\end{aligned}
$$

Since $\operatorname{ker} U_{I_{0}}^{*}$ is not trivial, the claim follows.

\section{Metric InVARIANTS of Markov QUASI-Similarity}

By Proposition 1 the Markov quasi-similarity is stronger than spectral equivalence of Koopman representations (it will be clear from the results of this section that it is essentially stronger). In particular all spectral invariants like ergodicity, weak mixing, mild mixing, mixing and rigidity are invariants for Markov quasisimilarity. It also follows that each transformation which is spectrally determined, that is for which spectral equivalence is the same as measure-theoretical equivalence, is also Markov quasi-equivalence unique (up to measure-theoretic isomorphism). In particular each automorphism Markov quasi-similar to an ergodic transformation with discrete spectrum is isomorphic to it. The same holds for Gaussian-Kronecker systems (see [5]).

This spectral flavor is still persistent when we consider Markov quasi-images. Indeed, each Markov operator between $L^{2}$-spaces "preserves" the subspace of zero mean functions, therefore a direct consequence of (16) is that a transformation which is a Markov quasi-image of an ergodic (weakly mixing, mixing) system remains ergodic (weakly mixing, mixing). Despite all this, Markov quasi-similarity is far from being spectral equivalence. In order to justify this statement, we need a non-disjointness result from [17] (in fact its proof) which we now briefly recall.

Assume that $T_{i}$ is an ergodic automorphism of $\left(X_{i}, \mathcal{B}_{i}, \mu_{i}\right), i=1,2$ and let $\Phi: L^{2}\left(X_{1}, \mathcal{B}_{1}, \mu_{1}\right) \rightarrow L^{2}\left(X_{2}, \mathcal{B}_{2}, \mu_{2}\right)$ be a Markov operator intertwining $U_{T_{1}}$ and 
$U_{T_{2}}$. Then $\Phi$ sends $L^{\infty}$-functions to $L^{\infty}$-functions and we can consider $H_{\Phi}$, the $L^{2}$-span of

$$
\left\{\Phi\left(f_{1}^{(1)}\right) \cdot \ldots \cdot \Phi\left(f_{m}^{(1)}\right): f_{i}^{(1)} \in L^{\infty}\left(X_{1}, \mathcal{B}_{1}, \mu_{1}\right), i=1, \ldots, m, m \geq 1\right\} .
$$

It turns out that $H_{\Phi}=L^{2}\left(\mathcal{A}_{\Phi}\right)$ where $\mathcal{A}_{\Phi} \subset \mathcal{B}_{2}$ is a $T_{2}$-invariant $\sigma$-algebra (in other words $\Phi$ defines a factor of $T_{2}$ ). Then by the proof of the main non-disjointness result (Theorem 4) in [17] this factor is also a factor of an (ergodic) infinite selfjoining of $T_{1}$. If we assume additionally that $\operatorname{Im} \Phi$ is dense then $H_{\Phi}=L^{2}\left(X_{2}, \mathcal{B}_{2}, \mu_{2}\right)$ and the factor given by $\mathcal{A}_{\Phi}$ is equal to $T_{2}$ itself.

Proposition 8. If $T_{2}$ is a Markov quasi-image of $T_{1}$ then $T_{2}$ is a factor of some infinite ergodic self-joinings of $T_{1}$.

As all the systems determined by (infinite) joinings of zero entropy systems have zero entropy and the systems given by joinings of distal systems are also distal (for these results see e.g. [7]), Proposition 8 yields the following conclusion.

Proposition 9. Each automorphism which is a Markov quasi-image of a zero entropy system has zero entropy. Each automorphism which is a Markov quasiimage of a distal system remains distal. In particular, zero entropy and distality are invariants of Markov quasi-similarity in the class of measure-preserving systems. $\square$

As a matter of fact, we can prove that zero entropy is an invariant of Markov quasi-similarity in the class of measure-preserving systems in a simpler manner. Recall that $T_{1}$ and $T_{2}$ are said to be disjoint (in the sense of Furstenberg [6]) if the only joining between them is the product measure. The following result will help us to indicate further invariants of Markov quasi-similarity.

Lemma 10. If $T_{1}$ is disjoint from $S$ and $T_{2}$ is a Markov quasi-image of $T_{1}$ then $\mathrm{T}_{2}$ is also disjoint from $S$.

Proof. Indeed, assume that $\Phi \circ U_{T_{1}}=U_{T_{2}} \circ \Phi$ and $\Phi$ has dense range. If $T_{2}$ and $S$ are not disjoint then we have a non-trivial Markov operator $\Psi$ intertwining $U_{T_{2}}$ and $U_{S}$. Since $\Phi$ has dense range, $\Psi \circ \Phi$ is a non-trivial Markov operator intertwining $U_{T_{1}}$ and $U_{S}$ and therefore $T_{1}$ is not disjoint from $S$.

Given a class $\mathcal{M}$ of automorphisms denote by $\mathcal{M}^{\perp}$ the class of those transformations which are disjoint from all members of $\mathcal{M}$. In view of Lemma 10 we have the following.

Proposition 11. $\mathcal{M}^{\perp}$ is closed under taking automorphisms which are Markov quasi-images of members of $\mathcal{M}^{\perp}$. In particular, if $\mathcal{M}=\mathcal{M}^{\perp \perp}$ then $\mathcal{M}$ is closed under taking automorphisms which are Markov quasi-images of members of $\mathcal{M}$.

If by $\mathcal{K}$ and $\mathcal{Z E}$ we denote the classes of Kolmogorov automorphisms and zero entropy automorphisms respectively then we have $\mathcal{K}=\mathcal{Z E}^{\perp}([6])$ and therefore by Proposition 11 we obtain the following.

Corollary 12. Every automorphism which is a Markov quasi-image of a Kolmogorov automorphism is also $K$. In particular, $K$ property is an invariant of Markov quasi-similarity in the class of measure-preserving systems.

Problem 1. Is the same true for Bernoulli automorphisms?

Notice that also $\mathcal{Z E}=\mathcal{K}^{\perp}$. Therefore we can apply Proposition 11 with $\mathcal{M}=\mathcal{Z E}$ to obtain that an automorphism which is a Markov quasi-image of a zero entropy system has zero entropy. 


\section{JP PROPERTY AND MARKOV QUASI-SIMILARITY}

Definition. An ergodic automorphism $T$ on $(X, \mathcal{B}, \mu)$ is said to have the joining primeness (JP) property (see [16]) if for each pair of weakly mixing automorphisms $S_{1}$ on $\left(Y_{1}, \mathcal{C}_{1}, \nu_{1}\right)$ and $S_{2}$ on $\left(Y_{2}, \mathcal{C}_{2}, \nu_{2}\right)$ and for every indecomposable Markov operator

$$
\Phi: L^{2}(X, \mu) \rightarrow L^{2}\left(Y_{1} \times Y_{2}, \nu_{1} \otimes \nu_{2}\right)
$$

intertwining $U_{T}$ and $U_{S_{1} \times S_{2}}$ we have (up to some abuse of notation) $\operatorname{Im} \Phi \subset$ $L^{2}\left(Y_{1}, \mathcal{C}_{1}, \nu_{1}\right)$ or $\operatorname{Im} \Phi \subset L^{2}\left(Y_{2}, \mathcal{C}_{2}, \nu_{2}\right)$.

The class of JP automorphisms includes in particular the class of simple systems ([10]). For other natural classes of JP automorphisms including some smooth systems see [16] (we should however emphasize that a "typical" automorphism is JP [16]).

Assume that $T$ is JP and $S_{1}, S_{2}, \ldots$ are weakly mixing. Let $\Phi: L^{2}(X, \mu) \rightarrow$ $L^{2}\left(Y_{1} \times Y_{2} \times \ldots, \nu_{1} \otimes \nu_{2} \otimes \ldots\right)$ be a Markov operator intertwining $U_{T}$ and $U_{S_{1} \times S_{2} \times \ldots}$. Let $\Phi=\int_{\Gamma} \Phi_{\gamma} d P(\gamma)$ be the decomposition corresponding to the ergodic decomposition of the joining determined by $\Phi$. Slightly abusing notation, we claim that for $P$-a.e. $\gamma \in \Gamma$

$$
\Phi_{\gamma}\left(L^{2}(X, \mathcal{B}, \mu)\right) \subset L^{2}\left(Y_{i_{\gamma}}, \mathcal{C}_{i_{\gamma}}, \nu_{i_{\gamma}}\right) \text {, for some } i_{\gamma} \in\{1,2, \ldots\} .
$$

Indeed, we use repeatedly the definition of JP property: We represent $\Pi_{n>1} S_{n}$ as $S_{1} \times\left(\Pi_{n \geq 2} S_{n}\right)$ and if $\operatorname{Im} \Phi_{\gamma}$ is not included in $L^{2}\left(Y_{1}, \nu_{1}\right)$ then $\operatorname{Im} \Phi_{\gamma} \subset L^{2}\left(Y_{2} \times Y_{3} \times\right.$ $\left.\ldots, \nu_{2} \otimes \nu_{3} \otimes \ldots\right)$. In the next step we write $\Pi_{n \geq 1} S_{n}=\left(S_{1} \times S_{2}\right) \times\left(\Pi_{n \geq 3} S_{n}\right)$ and we check if $\operatorname{Im} \Phi_{\gamma} \subset L^{2}\left(Y_{1} \times Y_{2}, \nu_{1} \otimes \nu_{2}\right)$ (if it is the case then $\operatorname{Im} \Phi_{\gamma} \subset L^{2}\left(Y_{2}, \nu_{2}\right)$ ); if it is not the case then $\operatorname{Im} \Phi_{\gamma} \subset L^{2}\left(Y_{3} \times Y_{4} \times \ldots, \nu_{3} \otimes \nu_{4} \otimes \ldots\right)$, etc. If for each $n \geq 1, \operatorname{Im} \Phi_{\gamma} \perp L^{2}\left(Y_{1} \times \ldots \times Y_{n}, \nu_{1} \otimes \ldots \otimes \nu_{n}\right)$, then $\operatorname{Im} \Phi_{\gamma}=0$ (since functions depending on finitely many coordinates are dense), and hence $\Phi_{\gamma}=0$.

It follows that for some $0 \leq a_{n} \leq 1$ with $\sum_{n \geq 1} a_{n}=1$

$$
\Phi=\sum_{n \geq 1} a_{n} \Phi_{n}
$$

where $\operatorname{Im} \Phi_{n} \subset L^{2}\left(Y_{n}, \mathcal{C}_{n}, \nu_{n}\right)$. In particular,

$$
\operatorname{Im} \Phi \subset \bigoplus_{n \geq 1} L^{2}\left(Y_{n}, \mathcal{C}_{n}, \nu_{n}\right) \subset L^{2}\left(Y_{1} \times Y_{2} \times \ldots, \mathcal{C}_{1} \otimes \mathcal{C}_{2} \otimes \ldots, \nu_{1} \otimes \nu_{2} \otimes \ldots\right)
$$

Note that the space $F:=\bigoplus L^{2}\left(Y_{n}, \nu_{n}\right)$ is closed and $U_{S_{1} \times S_{2} \times \ldots}$-invariant.

Lemma 13. Under the above notation, if $\mathcal{A} \subset \mathcal{C}_{1} \otimes \mathcal{C}_{2} \otimes \ldots$ is a factor of $S_{1} \times S_{2} \times \ldots$ and it is also a Markov quasi-image of a JP automorphism $T$ then there exists $n_{0} \geq 1$ such that $\mathcal{A} \subset \mathcal{C}_{n_{0}}$; in other words the factor given by $\mathcal{A}$ is a factor of $S_{n_{0}}$.

Proof. Asume that $\Phi$ intertwines $U_{T}$ and the Koopman operator of the factor action of $S_{1} \times S_{2} \times \ldots$ on $\mathcal{A}$. Since the range of $\Phi$ is dense in $L^{2}(\mathcal{A})$, it follows that $\Phi: L^{2}(X, \mathcal{B}, \mu) \rightarrow L^{2}(\mathcal{A}) \subset F$. We now use an argument from [9]: Take $A \in \mathcal{A}$. In view of (11) we have

$$
\mathbf{1}_{A}-\left(\nu_{1} \otimes \nu_{2} \otimes \ldots\right)(A)=f_{1}\left(y_{1}\right)+f_{2}\left(y_{2}\right)+\ldots
$$

with $f_{n} \in L_{0}^{2}\left(Y_{n}, \nu_{n}\right), n \geq 1$. Since the distribution of the random variable $\mathbf{1}_{A}-$ $\left(\nu_{1} \otimes \nu_{2} \otimes \ldots\right)(A)$ is a measure on a two element set and the random variables $f_{1}, f_{2}, \ldots$ are independent, all of them but one, say $f_{n_{A}}$, are equal to zero. In other 
words, $A \in \mathcal{C}_{n_{A}}$. It easily follows that the function $\mathcal{A} \ni A \mapsto n_{A}$ is constant (see [9]).

Let $T$ be a simple weakly mixing automorphism. By the definition of simplicity, it follows that each of its ergodic infinite self-joinings is, as a dynamical system, isomorphic to a Cartesian product $T^{\times n}$ with $n \leq \infty$. Since each simple system has the JP property, in view of Proposition 8 and Lemma 13 (in which $S_{n}=T$ ) we obtain the following.

Proposition 14. Each automorphism which is a Markov quasi-image of a simple map $T$ is a factor of $T$.

It follows from the above proposition that if $T_{1}$ and $T_{2}$ are weakly mixing simple automorphisms and are Markov quasi-similar then they are isomorphic.

Remark 3. In our example of $T_{1}$ and $T_{2}$ non-weakly isomorphic but Markov-quasisimilar $T_{2}$ is a factor of $T_{1}$ but (because of absence of weak isomorphism) $T_{1}$ is not a factor of $T_{2}$. Hence the family of factors of $T_{2}$ is strictly included in the family of automorphisms which are Markov quasi-images of $T_{2}$.

When we apply Proposition 14 to the MSJ maps (see [10]) we obtain that such systems are Markov quasi-similarly prime, that is we have the following.

Corollary 15. The only non-trivial automorphism which is a Markov quasi-image of an MSJ system $T$ is $T$ itself.

Remark 4. Assume that $T$ enjoys the MSJ property. Take $\Phi_{1}, \Phi_{2}$ two joinings of $T$ and $T \times T$ so that $\operatorname{Im} \Phi_{1} \cap\left(L^{2}(X, \mu) \otimes \mathbf{1}_{X}\right) \neq\{0\}$ and $\operatorname{Im} \Phi_{2} \cap\left(\mathbf{1}_{X} \otimes L^{2}(X, \mu)\right) \neq$ $\{0\}$. Then $\Phi:=a \Phi_{1}+(1-a) \Phi_{2}$ is a Markov operator intertwining $U_{T}$ and $U_{T \times T}$ and if $0<a<1$, then the range of $\Phi$ is not dense in $L^{2}\left(\mathcal{A}_{\Phi}\right)$. Indeed, $\mathcal{A}_{\Phi}$ is either $T \times T$ or $T \odot T$ (the factor of $T \times T$ determined by the $\sigma$-algebra of sets invariant under exchange of coordinates) and the claim follows from Lemma 13. This is the answer to a question raised by François Parreau in a conversation with the second named author of the note.

It means that if we try to define Markov quasi-image by requiring that $\mathcal{A}_{\Phi}=\mathcal{B}_{2}$ instead of requiring that the range of $\Phi$ is dense in $L^{2}\left(X_{2}, \mathcal{B}_{2}, \mu_{2}\right)$ then we obtain a strictly weaker notion.

\section{Final Remarks and problems}

Notice that the joining of $T_{1}$ and $T_{2}$ corresponding to the Markov operator in Section 3 and based on constructions from 13 is not ergodic (i.e. the Markov operator is decomposable). In fact, in our construction of two non-weakly isomorphic Markov quasi-similar automorphisms $T_{1}$ and $T_{2}$ no Markov operator corresponding to an ergodic joining between $T_{1}$ and $T_{2}$ can have dense range. Indeed, first recall that ergodic Markov quasi-similar automorphisms have the same Kronecker factors. Then notice that $T_{1}$ and $T_{2}$ are compact abelian group extensions of the same (in [13] this is the classical adding machine system) Kronecker factor. Hence, assume that $T$ is an ergodic automorphism with discrete spectrum and let $\phi: X \rightarrow G$, $\psi: X \rightarrow H$ be ergodic cocycles with values in compact abelian groups $G$ and $H$ respectively. We then have the following.

$T_{\phi}$ and $T_{\psi}$ are Markov quasi-similar via indecomposable

Markov operators if and only if they are weakly isomorphic. 
Indeed, every ergodic joining between such systems is the relatively independent extension of the graph joining given by an isomorphism $I$ of so called natural factors $T_{\phi J}$ and $T_{\psi F}$ acting on $X \times G / J$ and $X \times H / F$ respectively, see [14]. The Markov operator $\Phi$ corresponding to such a joining is determined by the orthogonal projection on the $L^{2}\left(X \times H / F, \mu \otimes \lambda_{H / F}\right)$; in particular the range of $\Phi$ is closed. Therefore it has dense range only if $\operatorname{Im} \Phi=L^{2}\left(X \times H, \mu \otimes \lambda_{H}\right)$ which means that in fact $I$ settles a metric isomorphism of $T_{\psi}$ and a factor of $T_{\phi}$. In other words, $T_{\psi}$ is a factor of $T_{\phi}$.

This shows that there exist two ergodic automorphisms which are Markov quasisimilar but Markov quasi-similarity cannot be realized by indecomposable Markov operators with dense ranges.

We have been unable to construct an indecomposable 1-1 Markov operator $\Phi$ with dense range intertwining the Koopman operatros given by two non-isomorphic ergodic automorphisms $T_{1}$ and $T_{2}$. One might think about such a construction using Markov operators given as convex combinations of $U_{S_{i}}$ where $S_{i}$ are space isomorphisms which are not intertwining $T_{1}$ and $T_{2}$ (see e.g. [2] for the notion of near simplicity where similar idea is applied).

It seems that Proposition 11 rules out a possibility to find two Markov quasisimilar Gaussian automorphisms which are not isomorphic by a use of so called Gaussian joinings 17] (recall that Gaussian joinings are ergodic joinings). Indeed, once a Markov quasi-similarity is given by an integral of Markov operators corresponding to Gaussian joinings, it sends chaos into chaos (see 17 for details). In particular, first chaos is sent into first chaos, and we obtain quasi-similarity of the unitary actions restricted to the first chaos. By Proposition 1 these actions on the first chaos are spectrally equivalent which in turn implies measure-theoretic isomorphism of the Gaussian systems.

We do not know however if we can have two non-weakly isomorphic Poisson suspension systems which are Markov quasi-similar by a use of Poissonian joinings (which are ergodic), see [3] and [21].

Problem 2. Recall that in the construction carried out in Section $\left[T_{2}\right.$ was a factor of $T_{1}$. Is it possible to construct Markov quasi-similar automorphisms $T_{1}$ and $T_{2}$ such that $T_{1}$ and $T_{2}$ have no common (non-trivial) factors? Of course such $T_{1}$ and $T_{2}$ must not be disjoint (see [6]).

The most "popular" construction of a pair of non-disjoint systems without common factors is $(T, T \odot T)$ (for a particular $T$; see [9], 22]). Notice however that these two automorphisms are not Markov quasi-similar if $T$ has the JP property (see Lemma 13), that is, in all known cases where $T$ and $T \odot T$ have no common (isomorphic) non-trivial factors.

Problem 3. As we have already noticed in Remark 1, Markov quasi-affinity implies Markov quasi-similarity. Are these notions equivalent? If the answer is positive then each weakly isomorphic transformations would have to be Markov quasi-affine. Are examples of weakly isomorphic non-isomorphic automorphisms from [12], [13] or [22] Markov quasi-affine?

Problem 4. The examples of Markov quasi-similar automorphisms which are not isomorphic presented in this note have infinite spectral multiplicity. Is it possible to find such examples in the class of systems with simple spectrum (or of finite spectral multiplicity)? In the class of rank one systems? Recall that in case of 
finite spectral multiplicity systems their weak isomorphism implies isomorphism, see e.g. [18].

\section{ACKNOWLEDGEMENTS}

The authors would like to thank Vitaly Bergelson for fruitful discussions and stimulating questions on the subject. We would like also to thank Alexander Gomilko for his remarks on the content of Section 2.

\section{REFERENCES}

[1] H. Anzai, Ergodic skew product transformations on the torus, Osaka J. Math. 3 (1951), 83-99.

[2] A. Danilenko, On simplicity concepts for ergodic actions, J. Anal. Math. 102 (2007), 77-117.

[3] Y. Derriennic, K. Frạczek, M. Lemańczyk, F. Parreau, Ergodic automorphisms whose weak closure of off-diagonal measures consists of ergodic self-joinings, Coll. Math. 110 (2008), 81-115.

[4] C. Foiaş, B. Sz.-Nagy, Harmonic analysis of operators on Hilbert space, North-Holland Publishing Co., Amsterdam-London; American Elsevier Publishing Co., Inc., New York; Akadémiai Kiadó, Budapest 1970.

[5] C. Foiaş, S. Stratila, Ensembles de Kronecker dans la théorie ergodique, C.R. Acad. Sci. Paris, sr. A-B 267 (1968), A166-A168.

[6] H. Furstenberg, Disjointness in ergodic theory, minimal sets, and a problem of Diophantine approximation, Math. Systems Th. 1 (1967), 1-49.

[7] E. Glasner, Ergodic Theory via Joinings, Math. Surveys Monogr. 101, Amer. Math. Soc., Providence, RI, 2003.

[8] C. Hoffman, A K counterexample machine, Trans. Amer. Math. Soc. 351 (1999), 4263-4280.

[9] A. del Junco, M. Lemańczyk, Generic spectral properties of measure-preserving maps and applications, Proc. Amer. Math. Soc. 115 (1992), 725-736.

[10] A. del Junco, D. Rudolph, On ergodic actions whose self-joinings are graphs, Ergodic Theory Dynam. Systems 7 (1987), 531-557.

[11] A.B. Katok, J.-P. Thouvenot, Spectral Properties and Combinatorial Constructions in Ergodic Theory, Handbook of dynamical systems. Vol. 1B, 649-743, Elsevier B. V., Amsterdam, 2006.

[12] J. Kwiatkowski, M. Lemańczyk, D. Rudolph, Weak isomorphism of measure-preserving diffeomorphisms, Israel J. Math. 80 (1992), 33-64.

[13] M. Lemańczyk, Weakly isomorphic transformations that are not isomorphic, Probab. Theory Related Fields 78 (1988), 491-507.

[14] M. Lemańczyk, M.K. Mentzen, Compact subgroups in the centralizer of natural factors of an ergodic group extension of a rotation determine all factors, Ergodic Theory Dynam. Systems 10 (1990), 763-776.

[15] M. Lemańczyk, F. Parreau, Lifting mixing properties by Rokhlin cocycles, preprint.

[16] M. Lemańczyk, F. Parreau, E. Roy, Systems with simple convolutions, distal simplicity and disjointness with infintely divisible systems, preprint.

[17] M. Lemańczyk, F. Parreau, J.-P. Thouvenot, Gaussian automorphisms whose ergodic selfjoinings are Gaussian, Fund. Math. 164 (2000), 253-293.

[18] D. Newton, Coalescence and spectrum of automorphisms of a Lebesgue space, Z. Wahrscheinlichkeitstheorie und Verw. Gebiete 19 (1971), 117-122.

[19] D.S. Ornstein, Ergodic theory, randomness, and dynamical systems, James K. Whittemore Lectures in Mathematics given at Yale University. Yale Mathematical Monographs, No. 5. Yale University Press, New Haven, Conn.-London, 1974.

[20] W. Parry, Topics in ergodic theory, Cambridge Tracts in Mathematics, 75. Cambridge University Press, Cambridge-New York, 1981.

[21] E. Roy, Mesures de Poisson, infinie divisibilité et propriétés ergodiques, Thèse de doctorat de l'Université Paris 6 (2005).

[22] D. Rudolph, An example of a measure preserving map with minimal self-joinings, and applications, J. Analyse Math. 35 (1979), 97-122.

[23] V.V. Ryzhikov, Joinings, intertwining operators, factors and mixing properties of dynamical systems, Russian Acad. Izv. Math. 42 (1994), 91-114. 
[24] Ya.G. Sinai, On weak isomorphism of measure-preserving transformations, Mat. Sb. 63 (1964), 23-42; English translation: Transl. Amer. Math. Soc. 57 (1966), 123-143.

[25] A.M. Vershik, Polymorphisms, Markov processes, and quasi-similarity, Discrete and Continuous Dynam. Systems 13 (2005), 1305-1324.

K. Frączek and M. Lemańczyk, Faculty of Mathematics and Computer Science, Nicolaus Copernicus University, ul. Chopina 12/18, 87-100 Toruń, Poland

E-mail address: fraczek@mat.uni.torun.pl, mlem@mat.uni.torun.pl 